\title{
Determination Of Newtonian Gravitation From The Schwarzschild Spacetime Within The Region Of Spherical Geometry
}

\author{
${ }^{1}$ Okpara, P. A., ${ }^{2}$ Oboma, D. N., Udeaja, V. N, ${ }^{4}$ Anih, J. O., \\ ${ }^{5}$ Onyia, A.I., ${ }^{6}$ Adieme, G.I., ${ }^{7}$ Nnachi N.O., ${ }^{2}$ Agha, S.O., ${ }^{2}$ Onah, D.U., \\ ${ }^{2}$ Agbo, P.E., ${ }^{2}$ Ekpe J.E., ${ }^{2}$ Anyigor, I. S. and ${ }^{2}$ Umahi, E. A. \\ ${ }^{I}$ Department of Industrial Mathematics and Applied Statistics, Ebonyi State UniversityAbakaliki,Nigeria. \\ ${ }^{2}$ Department of Industrial Physics Ebonyi State University, Abakaliki, Nigeria. \\ ${ }^{3}$ Department of Nursing Science, Ebonyi State University, Abakaliki, Nigeria. \\ ${ }^{4}$ Department of Physics, Federal College of Education Eha-amufu, Enugu State, Nigeria. \\ ${ }^{5}$ Department of Industrial Physics, Enugu State of Science and Technology, Enugu State, Nigeria. \\ ${ }^{6}$ Department of Science Lab. (Physics Option), Federal Polytechnic Oko, Anambara State, Nigeria. \\ ${ }^{7}$ Department of Science Education, Ebonyi State University, Abakaliki, Nigeria.
}

\begin{abstract}
General relativistic mechanics in gravitational fields exterior to homogenous spherically symmetric solution is developed using our coordinate displacement approach. In curved Schwarzschild spacetime, the coordinates provide a reference frame for an observer making measurements at an infinite distance from the gravitational source of the Schwarzschild spacetime. The Schwarzschild metric describes the spacetime surrounding a spherical non-rotating mass. However, physical quantities measured by arbitrary observers are not specified directly by these coordinates but rather are computed from the metric. It is well known that Newton's theory of gravity is an approximation to Einstein's theory when the curvature of space time is negligible. In this paper, we seek to deduce that in the limit of weak field approximation, Schwarzschild gravitation may be replaced with Newton's gravitation. Here, we present Schwarzschild gravitation and show its reduction to the classical Newtonian gravitation - using power series expansions.
\end{abstract}

Keywords: Gravitational field, General relativity, Schwarzschild spacetime, Newton's equations of motion.

\section{Introduction}

The Newton's dynamics theory suggested that all interactions of particles in nature manifest through force.This theory successfully explained the gravitational phenomena on earth and the experimental fact of the solar system. The Newton's dynamical laws of motion and gravitation are founded in terms of invariant rest masses of particles and bodies, thus cannot be applied to a photon which has no measurable rest mass (Chifu, 2008; Bergman, 1987; Chifu, 2010). At the end of the nineteenth century, there were several attempts to extend Newton's theory of gravitation to Einstein's gravitational field equations of motion in order to provide better agreement with the experimental data or better consistency to all physical theories. In 1915, Einstein published his geometrical theory of gravitation which is popularly known as general relativity (Misner et al., 1973).The General relativity tries to describe the gravitational field and the motion of test particles in terms of a manifestation of the geometrical curving of space and time.This theory offered a resolution of the anomalous orbital precession as well as some gravitational phenomena involving light such as bending of light and the spectral shift by the Sun (Anderson, 1967b). The mathematical expression of the Einstein's field equation includes important two aspects: establishing the theory of solutions and finding exact solution with physical background. Upto now, very few results on the theory of solutions for the Einstein's field equations have been established. The exact solutions are very helpful to understand the theory of general relativity and the universe. Typical examples of exact solutions are the Schwarzschild solution and Kerr solution. These solutions provide two important physical spacetimes: the Schwarzschild solution describes a stationary, spherically symmetric and asymptotically flat spacetime, while Kerr solution provides a stationary, axisymmetric and asymptotically flat spacetime. The investigation on exact solutions of the Einstein's field equations has debatable long history. In December, 1915, Schwarzschild discovered the first nontrivial solution to the vacuum Einstein's field equations which is a static solution with zero angular momentum (DeXing and KeFeng, 2010; Gruberetal, 1988 and Markley, 1973).

After Einstein's theory of special relativity which elegantly describes mechanics in electromagnetic and empty spaces, Einstein expected gravitation to have the same nature as electromagnetism and hence fit into 
special relationship. In special relativity, spacetime has four dimensions $(\alpha=0,1,2,3)$ and there always exist a global coordinate system in which the world-line element takes the form.

$$
c^{2} d \tau^{2}=n_{\alpha \beta} d x^{\alpha} d x^{\beta}
$$

where $n_{\alpha \beta}$ is a special relativistic metric tensor given by $n_{00}=1, n_{11}=n_{22}=n_{33}=-1\left(n_{\alpha \beta}=0, \alpha \neq\right.$ $\beta$ Such a coordinate system is said to be Cartesian. In a non-Cartesian coordinate system such as a spherical or spheroidal coordinates, the world-line element of space time may be expressed as

$$
c^{2} d \tau^{2}=g_{\alpha \beta} d x^{\alpha} d x^{\beta}
$$

where $g_{\alpha \beta}$ is the corresponding metric tensor which is generally different from the Cartesian metric tensor $n_{\alpha \beta}$. In practical calculations, the metric is most often written in coordinates which it takes the following form

$$
d s^{2}=g_{\alpha \beta} d x^{\alpha} d x^{\beta}
$$

According to the philosophy of general relativity, the effect of gravitation contain the metric tensor field $g_{\alpha \beta}$. Therefore, Einstein's theory of gravity, the gravitational field is promoted to a spacetime metric $g_{\alpha \beta}$.

Einstein's physical intuition motivated formulation of special relativity.but, the generalization to general relativity would not have occurred without the mathematical formulation given by Hermann Minkowski in 1909 (Synge 1960). The Minkowski metric was originally derived based on Hermann Minkowski's fundamental axiom for spacetime set out in an address given in September 1908: the substance at any worldpoint may always, with the appropriate determination of space and time, be looked as at rest in space time (Ref........). Therefore,Minkowski took the three spatial dimensions with an absolute time manifold that represented spacetime. Thus, the Minkowski metric appears in Cartesian coordinate as given by

$$
d s^{2}=-c^{2} d \tau^{2}+d x^{2}+d y^{2}+d z^{2}
$$

Eq.4 is arranged to provide information useful to obtain values of time coordinate of the local reference frame from the reference coordinate $(\mathrm{x}, \mathrm{y}, \mathrm{z}, \mathrm{t})$. The Cartesian coordinates used to express the Minkowski metric can also be converted to Minkowski metric spherical coordinates which have the form

$$
d s^{2}=-c^{2} d \tau^{2}+d r^{2}+r^{2} d \theta^{2}+r^{2} \sin ^{2} \theta d \emptyset^{2}
$$

The mathematical description of general relativity is given by Einstein's field equations (Einstein, 1916), which includes a metric tensor $g_{\alpha \beta}$ containing the information about the curvature of spacetime, or in other words how distances have been measured. One of the simplest, but nevertheless most important solution of Einstein's field equations is the Schwarzschild metric. The Schwarzschild metric is the unique spherically symmetric vacuum solution for Einstein's field equations formally by Schwarzschild (1916). In the spherical coordinates $x^{\mu}=(t, r, \theta, \emptyset)=(t, x, y, z)$ the line element is given by

$$
d s^{2}=-\left(1-\frac{2 M}{r}\right) d t^{2}+\left(1-\frac{2 M}{r}\right)^{-1} d r^{2}+r^{2}\left(d \theta^{2}+\sin ^{2} \theta d \phi^{2}\right)
$$

Several authors have published works in line with the research: Thesolutions of the Einstein Equation and General Relativity (Anderson, 1967a and 1967b,Bergamann, 1987); The static solutions of Einstein's Field Equations (EFE) for charged spheres of fluid (Nduka, 1978); TheSchwarzschild Black Hole (SBH) as a gravitational mirror (Stuckey,1993 ); The motion in the Schwarzschild Metric (Markley, 1973); The dynamical system's approach to Schwarzschild null geodesics (Belbruno and Pretorius, 2011); The motion of test particles and orbits exterior to static homogenous prolatespheroidal spacetime (Chifu, 2010); The general relativistic equations of motion for test particles exterior to astrophysically real or hypothetical spherical distributions of mass whose tensor field varies with azimuthal angle(Chifu and Lucas, 2008), The time-periodic solutions of the Einstein's field equations ( Dexing and Kefeng, 2010); The impossibility of a simple derivation of the Schwarzschild metric (Gruber et al.,1988); TheRiemannian Laplacian in Cartesian coordinate using great metric tensor of all gravitational fields in nature in Cartesian coordinate as the Fundamental Quantities of Riemannian Geometry (Omonile, et al., 2015).In this work, the determination of Newtonian gravitation from the schwarzschildspacetime far away from the gravitating compact massis investigated using power series expansion.

\section{Method}

The Schwarzschild metric, describing a non-rotating blackhole of mass $\mathrm{M}$, has the following line element in standard (spherical polar) Schwarzschild coordinates:

$$
\begin{gathered}
d s^{2}=g_{\alpha \beta} d x^{\alpha} d x^{\beta} \\
d s^{2}=-\left(1-\frac{2 M}{r}\right) d t^{2}+\left(1-\frac{2 M}{r}\right)^{-1} d r^{2}+r^{2}\left(d \theta^{2}+\sin ^{2} \theta d \phi^{2}\right)
\end{gathered}
$$

In order to determine the radial distance between two concentric circles in the Schwarzschild geometry, we set $(\mathrm{t}, \theta, \varnothing)$ to be a constant, such that we have

$$
d t=d \theta=d \emptyset=0
$$


Substituting equation (8) into equation (7)

$$
d s^{2}=\left(1-\frac{2 M}{r}\right)^{-1} d r^{2}
$$

Then equation (9) becomes

$$
d s=\left(1-\frac{2 M}{r}\right)^{-1 / 2} d r
$$

We have to restrict equation (10) to the null case in which $\mathrm{ds}=0$, that is the interval is a null curve

The solution to equation (11) becomes

$$
\left(1-\frac{2 M}{r}\right)^{-1 / 2} d r=0
$$

$$
d r \neq 0
$$

It has been known since 1916 that it is possible for a spherical body to have a point outside it at which the Schwarzschild metric has a singularity. This singularity is denoted by $\gamma_{s}$ and is called the Schwarzschild singularity. It is given by the condition

$$
\left(1-\frac{2 M}{r_{s}}\right)=0
$$

Thus

$$
r_{s}=2 M
$$

For most physical bodies in the universe, the Schwarzschild radius is much smaller than the radius of their surface. It is however, speculated that there exist some bodies in the universe with the Schwarzschild radius in the exterior region.

\section{Theory}

Let us consider Schwarzschild gravitation which is a solution of Einstein's theory of relativity as an improvement to Newtonian gravitation. The line element of Schwarzschild metric is given by

$$
d s^{2}=-\left(1-\frac{2 G M}{r c^{2}}\right)(c d t)^{2}+\left(1-\frac{2 G M}{r c^{2}}\right) d r^{2}+r^{2} d \theta^{2}+r^{2} \sin ^{2} \theta d \phi^{2}
$$

The metric given in equation (15) can be represented by

$$
d s^{2}=-e^{\frac{2 \phi}{c^{2}}}(c d t)^{2}+(d l)^{2}
$$

$d$ lin equation (16) represents the spatial part of the metric. The relativistic gravitational potential $\phi$ is obtained by the comparison of equations (15) and (16) as

$$
-\left(1-\frac{2 G M}{r c^{2}}\right)(c d t)^{2}=-e^{\frac{2 \phi}{c^{2}}}(c d t)^{2}
$$

If we take $\phi=0$ in equation (16), then we recover Minkowski metric which is special relativity. We take the logarithm of equation (17) as

Therefore

$$
\operatorname{In}\left(1-\frac{2 G M}{r c^{2}}\right)=\frac{2 \phi}{c^{2}}
$$

$$
\phi=\frac{c^{2}}{2} \operatorname{In}\left(1-\frac{2 G M}{r c^{2}}\right)
$$

Equation (19) represents the exact expression of the relativistic gravitational potential equivalent to Schwarzschild curved spacetime. As the particle tends to move in flat space in equation (19), then the logarithm function can be expanded in power series. The logarithm expansion is given by

$$
\operatorname{In}(1+x)=x-\frac{1}{2} x^{2}+\frac{1}{3} x^{3}-\cdots \text { for }|x|<1
$$

The logarithm expansion in equation (20) is only valid for the weak gravitational field.

Let us substitute $x=-\frac{2 G M}{r c^{2}}$ into equation (20), then expansion of $\phi$ is obtained as

$$
\phi=-\frac{G M}{r}-\frac{1}{c^{2}}\left(\frac{G M}{r}\right)^{2}-\frac{4}{3} \frac{1}{c^{4}}\left(\frac{G M}{r}\right)^{2}-\cdots
$$

The first term in equation (21) represents the Newtonian gravitational potential $\phi=-\frac{G M}{r}$. It shows that the Newtonian gravitation equilibrate the first term in otherwise curved spacetime in Schwarzschild metric. In this case, the Schwarzschild metric can be reduced to a corresponding Newtonian gravitation. We can reduce the coordinate distance in the metric by power series expansion using binomial expansion as

$$
(1+x)^{n}=1+\frac{n}{1 !} x+\frac{n(n-1)}{2 !} x^{2}+\cdots
$$

We expand the term $\left(1-\frac{2 G M}{r c^{2}}\right)^{-1}$ using power series expansion where $\mathrm{n}=-1, x=\frac{-2 G M}{r c^{2}}$

$$
\left(1-\frac{2 G M}{r c^{2}}\right)^{-1}=1+\frac{2 G M}{r c^{2}}+\frac{4}{c^{4}}\left(\frac{G M}{r}\right)^{2}+\cdots
$$

The first two terms of equation (23) is given by 


$$
\left(1-\frac{2 G M}{r c^{2}}\right)^{-1} \cong 1+\frac{2 G M}{r c^{2}}
$$

Minkowski's metric in spherical coordinates $r, \theta$ and $\emptyset$ is given by

$$
d s^{2}=-c^{2} d t^{2}+d r^{2}+r^{2} d \theta^{2}+r^{2} \sin ^{2} d \emptyset
$$

The metric in equation (25) can be written in matrix form as

$$
g_{\alpha \beta}=\left[\begin{array}{cccc}
-1 & 0 & 0 & 0 \\
0 & 1 & 0 & 0 \\
0 & 0 & r^{2} & 0 \\
0 & 0 & 0 & r^{2} \sin ^{2} \theta
\end{array}\right]
$$

Substituting the first two terms of equation (23) into the diagonal of Minkowski metric tensor in equation (26) then we have

$$
g_{\alpha \beta}=\left[\begin{array}{cccr}
-1+\frac{2 G M}{r c^{2}} & 0 & 0 & 0 \\
0 & 1+\frac{2 G M}{r c^{2}} & 0 & 0 \\
0 & 0 & r^{2} & 0 \\
0 & 0 & 0 & r^{2} \sin ^{2} \theta
\end{array}\right]
$$

Considering equation (27), the Schwarzschild metric becomes slightly curved and corresponds to Newtonian gravitation. Let us consider a coordinate with the metric tensor field. The weakness of the gravitational field allows us to decompose the metric tensor into the Minkowski metric plus a small perturbation as given below:

$$
g_{\alpha \beta}=\eta_{\alpha \beta}+h_{\alpha \beta},\left|h_{\alpha \beta}\right| \ll 1
$$

Equation (24) is equivalent to equation (28) which is substituted to equation (26) to become equation (27) as shown above in equation (27).

We have determined a Minkowski metric from a Schwarzschild metric with a perturbation $\frac{2 G M}{r c^{2}}$ as given in equation (27).

It is observed that for a single gravitating body, we will recover the Newtonian gravitation as shown in equation (21) given by

$$
\phi=-\frac{G M}{r}
$$

We have determined that Newtonian gravitation can be expressed in terms of a curved spacetime. It shows that Newtonian gravitation corresponds to Schwarzschild spacetime modified to be just slightly curved, which occurs far away from the gravitational source as described by equation (27). We have shown that the curvature of spacetime is sufficient to describe gravity in the Newtonian limit as given in equations (27) and (29).

\section{Conclusion}

In this paper, we have investigated the relationship between Newton's gravitation and Schwarzschild gravitation. We have been able to show that Newtonian gravitation can be expressed in terms of a curved spacetime. We have shown that Newtonian gravitation corresponds to Minkowski'sspacetime slightly modified to be flat and also Newtonian gravitation corresponds to Schwarzschild's spacetime modified to be just slightly curved, which occurs far away from the gravitational source (the mass M). Thus Schwarzschild spacetime is asymptotically flat in the region of large radius. This is what one might expect physically when one gets far away from the source of gravity.

\section{References}

[1]. Anderson, J. L. (1967a). Solutions of the Einstein Equation. In: Principles of Relativity Physics. Academic Press, New York, 372 402.

[2]. Anderson, J. L. (1967b). Experimental Test of General Relativity. In: Principles of Relativity Physics. Academic Press, New York $403-421$.

[3]. Belbruno, E. and Pretorius, F. (2011). A dynamical System's Approach to Schwarzschild null Geodesics. Classical and Quantum Gravity, 28(2011) 195007.

[4]. Bergamann, P. G. (1987). Introduction to the Theory of Relativity. Prentice Hall. $203-215$.

[5]. Chifu E.N. (2010). Motion of Test Particles and Orbits Exterior to Static Homogenous Prolate Spheroidal Spacetime. The African Physical Review, Vol4, $113-118$

[6]. Chifu, E.N. and Lucas, W.L. (2008). General Relativistic Equations of Motion for Test Particles Exterior to Astrophysically Real or Hypothetical Spherical Distributions of Mass whose Tensor Field Varies with Azimuthal Angle only. Continental Journal of Applied Sciences, 3(2): $32-38$.

[7]. Dexing, K. and Kefeng, L. (2010). Time-periodic Solutions of the Einstein's Field Equations I: General Framework. Science China Mathematics 53(5): 1213 - 1230. 
[8]. Gruber, R.P., Price, R.H., Matthews, S.M., Cordwell, W.R. and Wagner, L.F. (1988). The impossibility of a simple derivation of the Schwarzschild metric. Am. J. Phys. 56(3): 265 - 269.

[9]. Markley, F.L. (1973). Motion in the Schwarzschild Metric. AJP, Vol. 41: $45-50$.

[10]. Misner, K.S., Thorne,A.S. and Wheeler,J.A. (1973) Gravitation: Freeman, San Francisco Cambridge University Press.

[11]. Nduka, A. (1978). Static Solutions of Einstein's Field Equations for Charged Spheres of Fluid. ACTA PHYSICA POLONICA, VolB9(7).

[12]. Omonile, J.F; Kaffa, D.J. and Howusu, S.X.K. (2015). Riemannian Laplacian in Cartesian coordinate using great metric tensor of all gravitational fields in nature in Cartesian coordinate as the Fundamental Quantities of Riemannian Geometry. African Journal of Science and Research, 4(4): $1-3$.

[13]. Stuckey, W.M. (1993). Schwarzschild Black Hole as a Gravitational Mirror. Am. J. Phys, 61(5).

[14]. Synge, J.L. (1960) Relativity: The General Theory (North Holland Amsterdam) 